\title{
Neurons in the dorsomedial hypothalamus promote, prolong, and deepen torpor
}

\section{Michael Ambler ${ }^{1}$, Timna Hitrec ${ }^{1}$, Andrew Wilson ${ }^{1}$, Matteo Cerri ${ }^{2}$, Anthony Pickering ${ }^{1}$}

1. Anaesthesia, Pain, \& Critical Care Sciences, Department of Physiology, Pharmacology, \& Neuroscience, University of Bristol, UK

\section{Department of Biomedical \& Neuromotor Sciences, University of Bologna, Italy}

\section{Abstract}

Torpor is a naturally occurring, hypometabolic, hypothermic state engaged by a wide range of animals in response to imbalance between the supply and demand for nutrients. Recent work has identified some of the key neuronal populations involved in daily torpor induction in mice, in particular projections from the preoptic area of the hypothalamus (POA) to the dorsomedial hypothalamus (DMH). The DMH plays a role in thermoregulation, control of energy expenditure, and circadian rhythms, making it well positioned to contribute to the expression of torpor. We used activity dependent genetic TRAPing techniques to target $\mathrm{DMH}$ neurons that were active during natural torpor bouts in female mice. Chemogenetic reactivation of torpor-TRAPed DMH neurons in calorie-restricted mice promoted torpor, resulting in longer and deeper torpor bouts. Chemogenetic inhibition of torpor-TRAPed DMH neurons did not block torpor entry, suggesting a modulatory but not a necessary role for the $\mathrm{DMH}$ in the control of torpor. This work adds to the evidence that a projection from the POA to the $\mathrm{DMH}$ forms part of a torpor-inducing circuit within the mouse hypothalamus.

\section{Significance}

Daily heterotherms such as mice employ torpor to cope with environments in which the supply of metabolic fuel is not sufficient for the maintenance of normothermia. Daily torpor involves reductions in body temperature, as well as active suppression of heart rate and metabolism. How the central nervous system controls this profound deviation from normal homeostasis is not known, but a projection from the preoptic area to the dorsomedial hypothalamus has recently been implicated. We demonstrate that the dorsomedial hypothalamus contains neurons that are active during torpor. Activity in these neurons promotes torpor entry and maintenance but is not necessary for torpor entry. 
Torpor is the naturally occurring hypothermic, hypometabolic and hypoactive component of hibernation, which can be prolonged (in seasonal hibernators), or brief (in daily heterotherms such as the mouse). It serves as an adaptive response to relative energy deficit: a controlled reduction in metabolic demand in response to reduced availability of substrate. During torpor, body temperature typically runs a few degrees above ambient temperature, which in hibernating arctic ground squirrels results in core temperatures as low as $-2.9^{\circ} \mathrm{C}$ (Barnes, 1989). Metabolic rate falls to between 1 and $5 \%$ of euthermic rates with similar reductions in heart and respiratory rates (Heldmaier, Ortmann and Elvert, 2004).

The mechanisms controlling this profound deviation from normal physiology are not known, but recent work has indicated a role for the preoptic area of the hypothalamus (POA) in generating a hypothermic, hypometabolic, bradycardic state similar to daily torpor in the mouse (Hrvatin et al., 2020; Takahashi et al., 2020; Zhang et al., 2020). These neurons are active during natural fastinginduced daily torpor (Hrvatin et al., 2020; Zhang et al., 2020); are necessary for the full expression of daily torpor (Hrvatin et al., 2020; Takahashi et al., 2020; Zhang et al., 2020); express Adcyap1 (Hrvatin et al., 2020), and/or pyroglutamylated RFamide peptide (QRFP) (Takahashi et al., 2020), and/or oestrogen receptors (Zhang et al., 2020) with evidence of overlap in these markers. These studies suggest that activation of a projection from the POA to the dorsomedial hypothalamus $(\mathrm{DMH})$ contributes to the generation of this torpor-like state, although projections from the POA to arcuate may also contribute (Hrvatin et al., 2020; Zhang et al., 2020).

Projections from the POA to DMH are also involved in thermoregulation outside of the context of torpor. Warm-activated neurons in the ventral part of the lateral POA (VLPO) project to the DMH and inhibit thermogenesis and locomotion (Zhao et al., 2017). Similarly, warm-activated neurons in the ventromedial POA (VMPO) that express Adcyap1 and brain-derived neurotrophic factor (BDNF) project to the DMH and suppress thermogenesis (Tan et al., 2016). These thermogenesis inhibiting warm-activated POA to $\mathrm{DMH}$ projections that play a role in thermoregulation are GABAergic (Tan et al., 2016; Zhao et al., 2017), in contrast to the predominantly glutamatergic projections that are implicated in torpor (Hrvatin et al., 2020; Takahashi et al., 2020). In addition to an established role in thermoregulation, the $\mathrm{DMH}$ also regulates energy balance and food intake (Yang et al., 2009; Bi, Kim and Zheng, 2012; Jeong et al., 2015a), heart rate (Piñol et al., 2018), and plays a role in adjusting circadian rhythms in response to food availability (Chou et al., 
2003; Gooley, Schomer and Saper, 2006; Mieda et al., 2006). Hence, the DMH is well positioned to play a role in the regulation of torpor, and there is evidence to support its activation during daily torpor in the mouse (Hitrec et al., 2019).

We hypothesised that the DMH plays a role in the control of torpor. To test this hypothesis we used activity-dependent targeting of neurons (DeNardo et al., 2019) within the DMH that were active during natural torpor bouts in female mice. Using this approach, we first labelled neurons within the hypothalamus that were active during torpor. Next, we expressed excitatory or inhibitory DREADDs (Roth, 2016) in DMH neurons that were active during torpor, and evaluated the effects of activating these receptors on the propensity to enter torpor during calorie restriction.

\section{Methods}

Mice

All studies had the approval of the local University of Bristol Animal Welfare and Ethical Review Board and were carried out in accordance with the UK Animals (Scientific Procedures) Act. Female TRAP2 (Allen et al., 2017; DeNardo et al., 2019), Ai14 (Madisen et al., 2010), and C57BL/6J mice, at least 8 weeks of age with mean body weight 22.9 (2.8) grams on experiment entry were used. Female mice were chosen because of their increased propensity to torpor (Swoap and Gutilla, 2009). Mice were maintained on a reversed 12 -hour light/dark cycle with lights off at 08:30, hence lights off was assigned as zeitgeber time (ZT) 0 . At all times mice had free access to water and standard mouse chow (LabDiet, St. Louis, MO 63144, USA) except during periods of calorie restriction when food was limited as detailed below. They were housed in groups of up to four.

A homozygous breeding colony was established from heterozygous TRAP2 mice kindly donated by the Liqun Luo laboratory in Stanford University, California. The strain is now available via Jackson laboratory (www.jax.org/strain/030323). The TRAP2 mouse line carries the gene for Cre-ERT2 under the fos promoter leading to Cre-ERT2 expression in active neurons. Homozygous Ai14 mice were obtained from the University of Bristol in-house colony, having been originally purchased from Jackson laboratories (www.jax.org/strain/007908). The Ai14 mouse carries a floxed gene encoding a red fluorophore (tdTomato) knocked into the Gt(ROSA)26Sor locus. This requires the action of Cre to remove a stop codon to allow tdTomato expression. Breeding pairs were established with homozygous TRAP2 and homozygous Ai14 mice to generate TRAP:Ai14 doubleheterozygous offspring. The resultant double-transgenic TRAP:Ai14 mice produce red fluorescent 
tdTomato protein in neurons that were active during the time period defined by the injection of 4hydroxytamoxifen (4-OHT).

\section{Viral vectors}

Three viral vectors were used:

AAV2-hSyn-DIO-hM3Dq-mCherry was a gift from Bryan Roth (Addgene viral prep \#44361-AAV2; www.addgene.org/44361) (Krashes et al., 2011); 4.6×1012 viral genome copies per ml. This vector delivered a Cre-dependent mCherry-tagged excitatory DREADD gene under the human synapsin promoter. It was mixed in a 4:1 ratio with the EGFP-expressing vector (used to identify the injection site), giving a final titre for this vector of $3.7 \times 10^{12}$ viral genomes per $\mathrm{ml}$.

AAV2-hSyn-DIO-hM4Di-mCherry was a gift from Bryan Roth (Addgene viral prep \#44362-AAV2; www.addgene.org/44362) (Krashes et al., 2011); 2×1013 viral genome copies per ml. This vector delivered a Cre-dependent mCherry-tagged inhibitory DREADD gene under the human synapsin promoter. It was mixed in a 1:2 ratio with the EGFP-expressing vector, and the resulting vector mixture further diluted in a 10-fold with sterile phosphate buffered saline, giving a final titre of $6.7 \times 10^{11}$ viral genomes per $\mathrm{ml}$.

pAAV2-CMV-PI-EGFP-WPRE-bGH was a gift from James M. Wilson (Addgene viral prep \#105530-

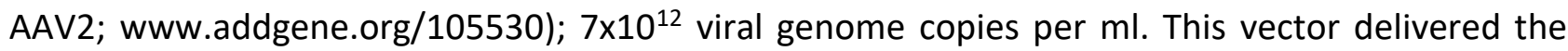
gene coding for enhanced green fluorescent protein (EGFP) under the ubiquitous CMV promoter. This vector was used to confirm the localisation of injection, because the expression of mCherry fluorescence in the two vectors above is contingent on successful TRAPing (and so would not be visible if the injected area is not TRAPed).

\section{Stereotaxic injection of viral vectors}

Mice were anaesthetised with ketamine $(70 \mathrm{mg} / \mathrm{kg}$ i.p.) and medetomidine $(0.5 \mathrm{mg} / \mathrm{kg}$ i.p.). Depth of anaesthesia was assessed and monitored by loss of hind paw withdrawal reflex. Additional i.p injections of anaesthetic were administered as needed to maintain surgical depth of anaesthesia. Core temperature was maintained using a servo-controlled heat pad and a rectal temperature probe (Harvard Apparatus). The planned incision site was shaved, and skin cleaned with iodine solution and sterile surgical technique was used throughout. Anaesthetised mice were placed in a 
stereotaxic frame, the head was fixed in atraumatic ear bars and skull position maintained horizontal by an incisor bar (David Kopf Instruments, USA).

Microcapillary pipettes were made from microcapillary glass (Sigma, USA) on a vertical pipette puller (Harvard Apparatus, UK). Pipettes were filled with mineral oil then vector was back-filled using a robotic microinjector (Nano-W wireless capillary microinjector, Neurostar, Germany), producing a visible vector-mineral oil interface. The scalp was incised in the midline and burr holes made bilaterally at bregma $-1.8 \mathrm{~mm}$, lateral $\pm 1 \mathrm{~mm}$ with a drill attachment (Neurostar, Germany). The microcapillary pipette was inserted at an angle of $8^{\circ}$ towards the midline. Bilateral injections were made at depths of 5 and $4.75 \mathrm{~mm}$ relative to the surface of the brain. Each injection was $180 \mathrm{nl}$ and was delivered at a rate of $100 \mathrm{nl} /$ minute. The injection pipette remained in place for one minute following the first injection and for five minutes after the second before removing.

Following vector injections, the wound was closed with non-absorbable suture and dressed with antibacterial wound powder. Anaesthesia was reversed with atipamezole $(1 \mathrm{mg} / \mathrm{kg}$ i.p, Antisedan, Zoetis). Buprenorphine was administered for analgesia (0.1mg/kg s.c., Vetergesic, Ceva Animal Health). Mice were recovered on a heat pad, then housed individually for three days following surgery and monitored daily until they recovered to baseline weight.

\section{Torpor induction}

Prior to torpor induction, mice were moved from the home cage to a custom built $32 \times 42 \times 56 \mathrm{~cm}$ cage, divided into four quadrants $(16 \times 21 \times 56 \mathrm{~cm})$, into which each mouse was individually placed. This cage was designed to allow up to 4 animals to be monitored simultaneously using a single thermal imaging camera placed directly above. The Perspex separating each quadrant was clear and had ventilation holes at $2 \mathrm{~cm}$ from the floor height to allow interaction between mice in neighbouring quadrants, while preventing huddling.

A food restriction protocol was used to increase the likelihood of torpor induction (see figure 1) (van der Vinne et al., 2018). Mice were weighed and given a single daily meal at lights off (ZTO) placed directly onto the floor of the cage for five consecutive days. The meal consisted of one pellet (2.2g) of feed (EUROdent Diet 22\%, irradiated, 5LF5). This provides $8 \mathrm{kcal}$ per day, which is approximately $70 \%$ of the estimated unrestricted daily intake for a mouse of this size (Subcommittee on Laboratory Animal Nutrition and Board on Agriculture, 1995). 
Mouse surface temperature was recorded using an infra-red thermal imaging camera placed above the cage (Flir C2, www.flir.co.uk). Baseline recordings of mouse surface temperature were taken for a period of three days at an ambient temperature of $21^{\circ} \mathrm{C}$. During this time, mice had free access to food and were therefore not expected to enter torpor. These measurements were then used to generate a daily profile mean and a standard deviation of the temperature fluctuations across the diurnal cycle (in one-minute bins). Torpor was defined as a period lasting at least 60 minutes during which surface temperature remained at least 4 standard deviations below the baseline at that circadian time (see figure $1 \mathrm{~A} \& \mathrm{~B}$ ).

\section{Experimental design and statistical analyses}

\section{TRAP:Ai14 tagging}

Female TRAP:Ai14 mice were entered into the calorie restriction protocol and were habituated to daily vehicle injection (chen oil, see below) at ZT7, before receiving 4-OHT on day four, again at ZT7. Approximately half the animals entered torpor within three hours of $4-\mathrm{OHT}(50 \mathrm{mg} / \mathrm{kg})$, but those that did not were used as controls.

\section{Chemogenetic targeting of $\mathrm{DMH}$ neurons}

Adeno-associated Viral vectors were used to deliver either Cre-dependent excitatory (hM3Dq ) or inhibitory (hM4Di) DREADD transgenes into the DMH of TRAP2 mice as described above. After at least two weeks recovery, mice were entered into the calorie restriction torpor induction protocol during which they were habituated to daily vehicle injection (chen oil, see below) at ZT7 before receiving 4-OHT on five, again at ZT7.

Following a further two weeks to allow return to baseline weight and to allow expression of the DREADD protein, mice entered a randomised, crossover design, calorie restriction trial. During this trial, each mouse was randomly assigned to receive either daily $\mathrm{CNO}(5 \mathrm{mg} / \mathrm{kg}$, i.p) or daily saline injections $(0.9 \%, 5 \mathrm{ml} / \mathrm{kg}, \mathrm{i} . \mathrm{p})$ at ZT7, on each of the five days of calorie restriction. The occurrence and depth of torpor was monitored with surface thermography. Following this first arm of the study, and after at least five days with free access to food, the process was repeated with mice that initially received CNO now receiving saline, and vice versa.

To control for a potential influence of CNO on torpor, wild type mice (without a vector injection) entered into the CNO vs saline crossover trial protocol. In order to control for an influence of 
basal DMH activity on torpor, further TRAP2 mice underwent DMH vector injection followed by 4OHT injection at ZT7 in the home cage with free access to food. These mice were then entered into the calorie restriction protocol with $\mathrm{CNO} /$ saline dosing. Torpor duration, and nadir temperature reached were recorded daily for each animal.

Mouse surface temperature was identified by extracting the maximum temperature value from regions of interest within each frame that correspond to each individual mouse's compartment within the cage. Peak temperature data was extracted from the infra-red video using Flir ResearchIR software version 4.40 .9 (www.flir.co.uk), and further filtered and analysed using Matlab 2019a (www.mathworks.com). The data processing stream was as follows: in order to limit noise and movement artefact, data points below 20 or above $40^{\circ} \mathrm{C}$ were removed; data was then interpolated and resampled at $1 \mathrm{~Hz}$, using the Matlab interp/ function; finally, a moving average filter function was applied with a 360 data point window, using the Matlab smooth function. Mouse activity data was derived from the thermal imaging video, extracted using Ethovision XT software (www.noldus.com).

\section{Histology: TRAP:Ai14 tagging}

Mice were culled by terminal anaesthesia (pentobarbitone (Euthatal) $175 \mathrm{mg} / \mathrm{kg}$,) a minimum of four weeks from the time of 4-OHT injection, to allow expression of tdTomato. They were transcardially perfused with $10 \mathrm{ml}$ heparinised $0.9 \%$ saline (50 units/millilitre) followed by $20 \mathrm{ml}$ of $10 \%$ neutral buffered formalin. Brains were removed and stored in fixative solution for 24 hours at $4^{\circ} \mathrm{C}$ before being transferred to $20 \%$ sucrose in $0.1 \mathrm{M}$ phosphate buffer (PB), $\mathrm{pH} 7.4$, and again stored at $4^{\circ} \mathrm{C}$. Brains were sectioned at $40 \mu \mathrm{m}$ thickness into a $1: 3$ series on a freezing microtome, transferred to $0.1 \mathrm{M}$ PB containing 1:1000 sodium azide. Sections were imaged using a Leica DMI6000 widefield microscope and 0.75 numerical aperture, 20-times magnification objective, excitation filter 546/10 nm, dichroic mirror 560nm, emission filter 580/40nm.

Masks for regions of interest were taken from the Mouse Brain Atlas (Franklin and Paxinos, 2007). These masks were then digitally applied to each of the widefield images so that the same size and shape area of interest was used for cell counting across animals. The $\mathrm{DMH}, \mathrm{PH}$, and Arc were defined by their atlas boundaries. The preoptic area mask was defined dorsally by the anterior commissure, ventrally by the ventral surface of the brain, and laterally by the lateral extent of the ventrolateral preoptic nucleus. Hence, the preoptic area as defined here included the medial and lateral parts of the medial preoptic nucleus, the ventromedial and ventrolateral preoptic nuclei, 
the medial and lateral preoptic areas, and parts of the strio-hypothalamic, the septohypothalamic, the median preoptic, and the periventricular nuclei (Franklin and Paxinos, 2007). An automated image processing pipeline was employed to count c-fos positive nuclei. This involved background subtraction with a rolling ball radius of 50 pixels. Labelled cells were identified by applying a threshold to the image that identified regions with brightness three standard deviations greater than the mean background. Overlapping regions were separated using the watershed method. Highlighted areas were then filtered by size (50-2000 $\left.\mu^{2}\right)$ and counted in Image-J.

\section{Histology: Chemogenetic targeting of DMH neurons}

Fresh frozen tissue was prepared by removal of the brain, which then was then mounted in optimal cutting temperature compound (www.fishersci.com) and drop-frozen in a metal container of isopentane on dry ice. Brains were stored at $-70^{\circ} \mathrm{C}$, then $15 \mu \mathrm{m}$ coronal sections were cut using a cryostat. Every third section was taken for immunohistochemistry (IHC) in order to confirm appropriate injection targeting and expression of the DREADD-mCherry fusion protein, which indicates successful TRAPing of cells. Sections were mounted onto glass slides (Superfrost Plus, ThermoFisher Scientific), and dried either overnight at $21^{\circ} \mathrm{C}$ or for 30 minutes at $30^{\circ} \mathrm{C}$. Sections were then blocked by incubation with $5 \%$ normal donkey serum in $0.3 \%$ Triton- $\mathrm{X}$ and $0.1 \mathrm{M}$ PB (PBT) for 4 hours. Primary mCherry primary (Biovision 5993, 1:2000) were diluted in 5\% normal donkey serum in PBT and applied to sections for overnight incubation at room temperature. After incubation in the primary antibody, sections were washed once with a $0.15 \%$ Triton-X in PB for 15 minutes, then in PB for a further 15 minutes. After washing, sections were incubated for 4 hours in donkey anti-rabbit secondaries (Alexafluor594, 1:1000). Sections were imaged using a Zeiss Axioskop II inverted microscope with a CooLED pE-100 190 excitation system, excitation filter 546/12nm, dichroic mirror 580nm, emission filter 590nm.

\section{Drug preparation}

\section{Vehicle}

The vehicle for 4-hydroxytamoxifen was chen oil, composed of four parts sunflower seed oil and one part castor oil.

\section{4-Hydroxytamoxifen}


The z-isomer of 4-hydroxytamoxifen (4-OHT) is the active isomer (www.tocris.com). It was dissolved in chen oil using the following method (Guenthner et al., 2013). First, 4-OHT was dissolved in neat ethanol at $20 \mathrm{mg} / \mathrm{ml}$ by shaking at $400 \mathrm{rpm}$ and $37^{\circ} \mathrm{C}$ for $30-60$ minutes until fully dissolved. Two parts chen oil for every one-part ethanol was then added, and the ethanol was evaporated off using a vacuum centrifuge leaving a final solution of $10 \mathrm{mg} / \mathrm{ml}$ in chen oil. Drug was prepared on the day of use, and if not used immediately, was kept in solution in the oil by shaking at $400 \mathrm{rpm}$ at $37^{\circ} \mathrm{C}$. Once in solution, the drug was protected from light.

\section{Clozapine-N-Oxide}

CNO was dissolved at $1 \mathrm{mg} / \mathrm{ml}$ in sterile water at room temperature. Aliquots were stored protected from light for up to one week at room temperature.

\section{Statistical analysis}

Data were analysed using GraphPad Prism (version 6.07). Data for time spent in torpor, nadir temperature, and cell count data were analysed using two-way repeated measures ANOVA with Holm-Sidak's multiple comparisons test. Analysis of the probability of torpor during calorie restriction in the absence of chemogenetic manipulation used Friedman (for repeated measures) or Kruskal-Wallis test with Dunn's multiple comparisons. Data for total number of days with torpor, day of first torpor entry, and weight at first torpor entry were analysed using paired samples $t$ test or Wilcoxon matched pairs signed rank test. Normality was assessed by Kolmogorov-Smirnov test. Data is presented as mean \pm standard deviation for normally distributed data, or else median [interquartile range]. Where the day of torpor emergence was analysed, if no bout appeared by the end of five days' calorie restriction, torpor was assumed to occur on day six. 


\section{Results}

\section{Torpor induction by calorie restriction}

Prior to chemogenetic manipulation of torpor-TRAPed neurons, the calorie restriction protocol generated torpor bouts on at least one day in $97 \%$ of 45 trials in 30 mice. Mean nadir surface temperature during torpor was $25.3 \pm 1.3^{\circ} \mathrm{C}$ compared to a mean nadir of $30.0 \pm 0.7^{\circ} \mathrm{C}$ in mice held at the same ambient temperature $\left(21^{\circ} \mathrm{C}\right)$ with free access to food $(t(11)=9.40, p<0.0001)$. Entry into torpor tended to occur in the second half of the lights off period, with the median time of entry into torpor occurring at ZT 9.76 [8.18 - 10.83] hours after lights off.

Torpor duration increased from median 1.27 hours [1.09 - 1.65] on day one to 4.47 hours [1.90 6.77] on day five (Kruskal-Wallis test, $H(5)=18.05, p<0.01$ ) (see figure 1E). The increase in torpor duration was associated with torpor occurring increasingly early in the day, from a median 12.42 hours [11.21 - 15.13] from lights off on day one to median 9.11 hours [7.87 - 10.77] on day five (Kruskal-Wallis $H(5)=20.20, p<0.001$ ). The nadir temperature reached during torpor decreased with increasing days of calorie restriction from $27.3 \pm 1.0^{\circ} \mathrm{C}$ on day one to $25.4 \pm 1.5^{\circ} \mathrm{C}$ on day five (one-way ANOVA $F(4,102)=4.717, p<0.01$ ) (see figure $1 F$ ). Activity of the mice, derived from the thermal imaging video, reduced to a minimum during torpor, and increased during arousal.

Mice lost weight across the five days of calorie restriction (see figure 1 C \& D). Torpor tended to emerge on day 3 [2-4], the probability of torpor increased with each day of calorie restriction, with $11.1 \%$ of trials resulting in torpor on day $1(95 \% \mathrm{Cl} 3.7$ to $24.1 \%)$ and $71.1 \%$ of trials resulting in torpor on day 5 (95\% $\mathrm{Cl} 55.7$ to $83.6 \%)$. Torpor was significantly more likely to occur when mouse weight reached $<23 \mathrm{~g}$, or weight loss was $>9 \%$ (see figure $1 \mathrm{G}-\mathrm{I}$ ).

\section{Torpor is associated with increased neural activity in the POA and DMH}

To identify neuronal populations that were active during torpor, we used calorie restriction to induce torpor in TRAP:Ai14 mice and administered 4-OHT $(50 \mathrm{mg} / \mathrm{kg}$ i.p) on the fourth day in in order to 'TRAP' active neurons. We observed widespread recombination resulting in tdTomato expression throughout the hypothalamus, both in mice that entered torpor (Torp+) following 4$\mathrm{OHT}$, and those that did not (Torp-, see figure 2).

Four hypothalamic areas were selected a-priori for cell counting: POA, DMH, posterior hypothalamus (PH), and arcuate nucleus (arc). Entry into torpor was associated with increased tdTomato labelled ('TRAPed') cells in the DMH and POA as compared to calorie-restricted mice 


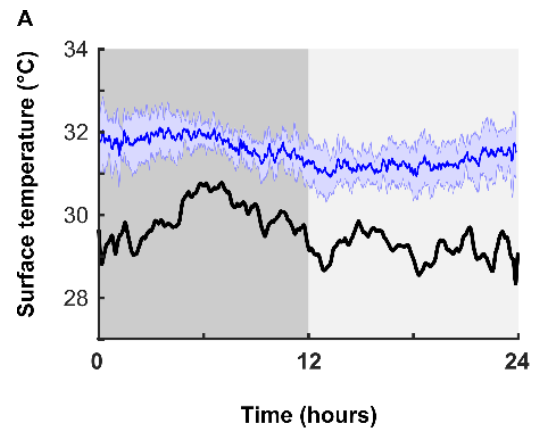

C
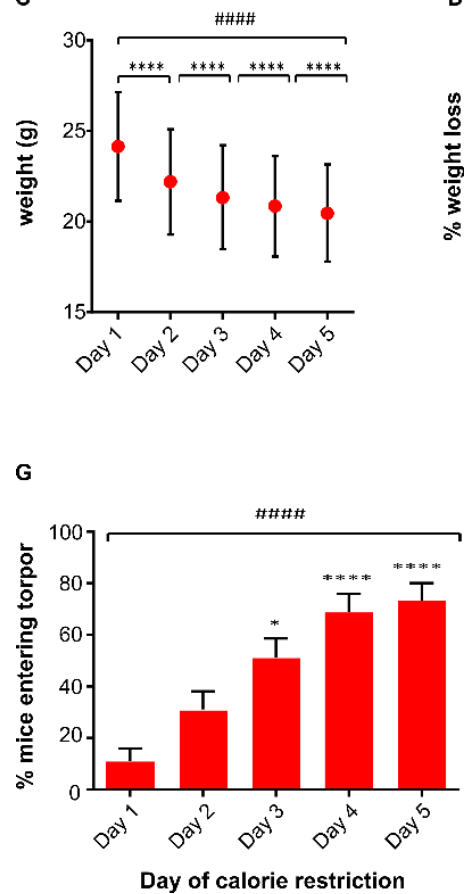
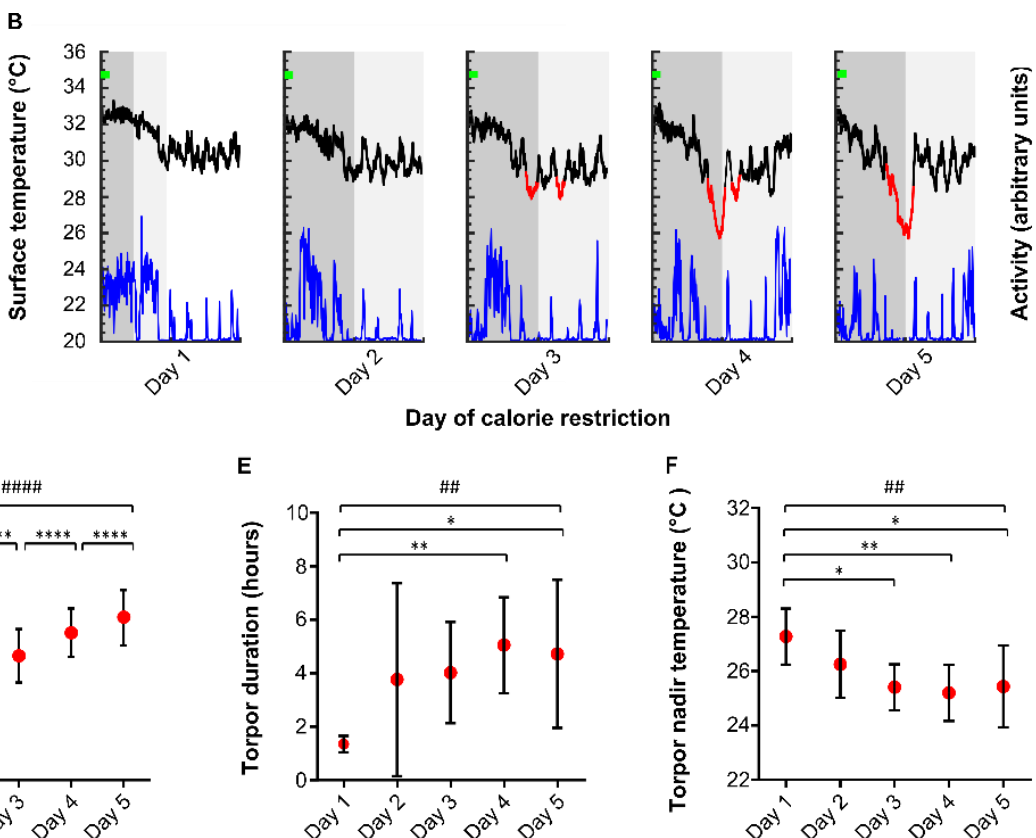

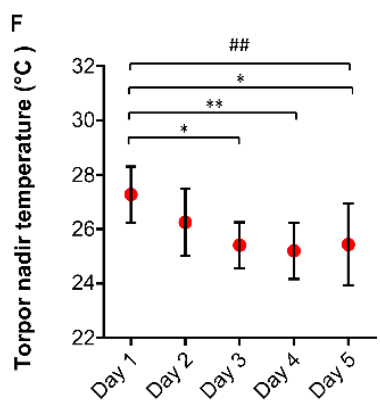

Day of calorie restriction

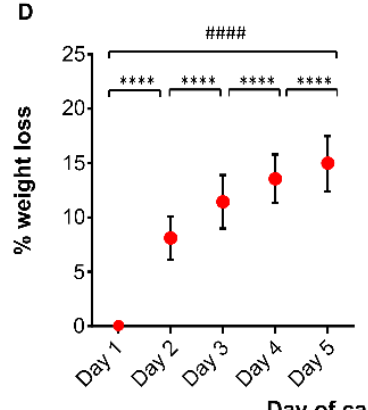

H

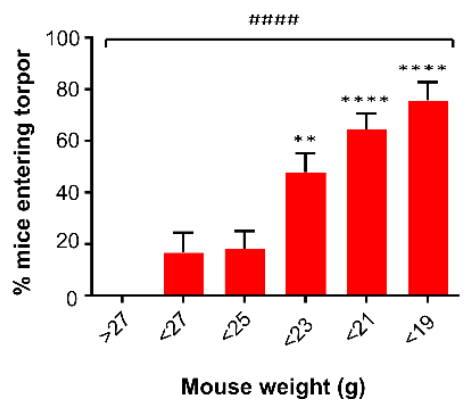

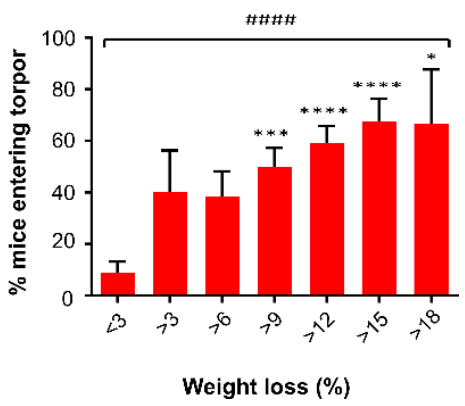

Figure 1. A, mean mouse surface temperature across the 24-hour day cycle housed at $21^{\circ} \mathrm{C}$ ( $\mathrm{n}=7$ female mice recorded for three consecutive days, blue line). Shaded area represents standard deviation for that zeitgeber time. Torpor threshold (black line) represents four standard deviations from the mean temperature at that zeitgeber time. $B$, example profile from single mouse undergoing torpor by 5 days calorie restriction. Mice receive a single meal at lights off (ZTO, green marker), providing approximately $70 \%$ of the ad-lib daily intake. Surface temperature (black / red line) is measured using infra-red thermography, activity (blue) derived from thermal imaging video. Torpor (red line) defined as a period during which surface temperature remained 4 standard deviations below the mean for that time of day for a period of at least one hour. C \& D, Weight loss by day of calorie restriction. E \& F, torpor bout duration and nadir surface temperature by day of calorie restriction. $G$, Proportion of mice entering torpor for each consecutive day of calorie restriction. $\mathrm{H}$, proportion of mice entering torpor by absolute weight. $\mathrm{H}$, proportion of mice entering torpor by \% weight loss from first day of calorie restriction. \#,\#\#,\#\#,\#\#\#\#= Main effect for day of calorie restriction or weight loss at $p<0.05,<0.01,<0.001$, or $<0.0001$, respectively. ${ }^{*}, * * * * *, * * * *$ indicate $p<0.05,<0.01,<0.001$, or $<0.0001$ respectively for individual comparisons. G-I individual comparisons are relative to \% mice entering torpor on day $1 . \mathrm{n}=45$ trials in 30 female mice, five consecutive days calorie restriction at $21^{\circ} \mathrm{C}$ ambient temperature with no chemogenetic manipulation. 
bioRxiv preprint doi: https://doi.org/10.1101/2021.09.05.458994; this version posted September 6, 2021. The copyright holder for this preprint (which was not certified by peer review) is the author/funder, who has granted bioRxiv a license to display the preprint in perpetuity. It is made available under aCC-BY-ND 4.0 International license.

A

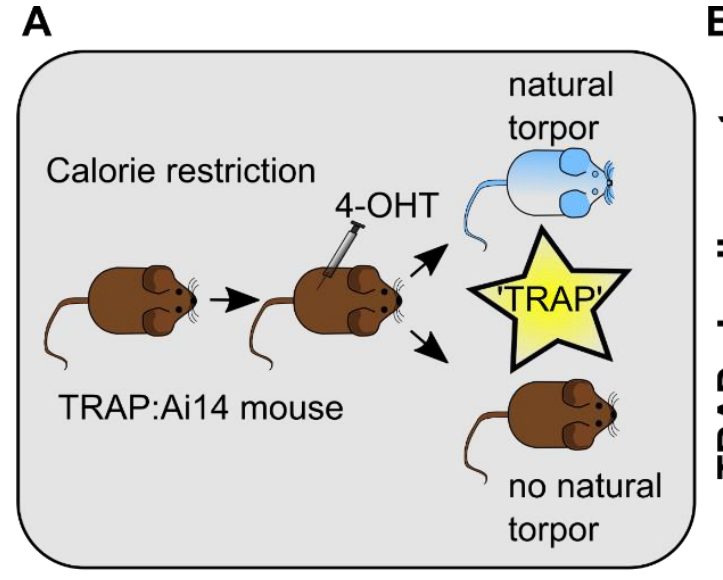

C

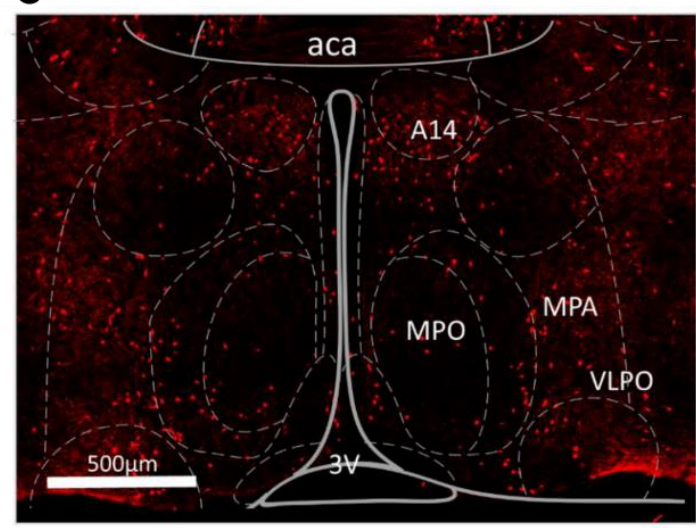

F

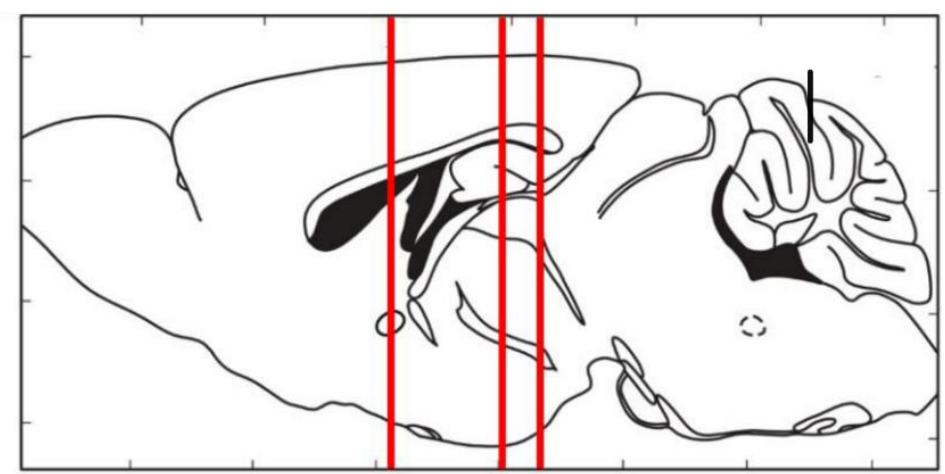

B

D
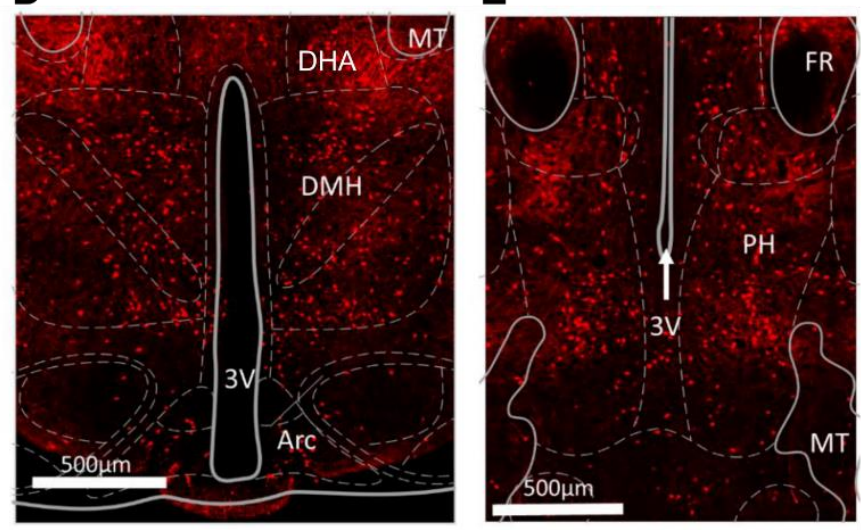

G

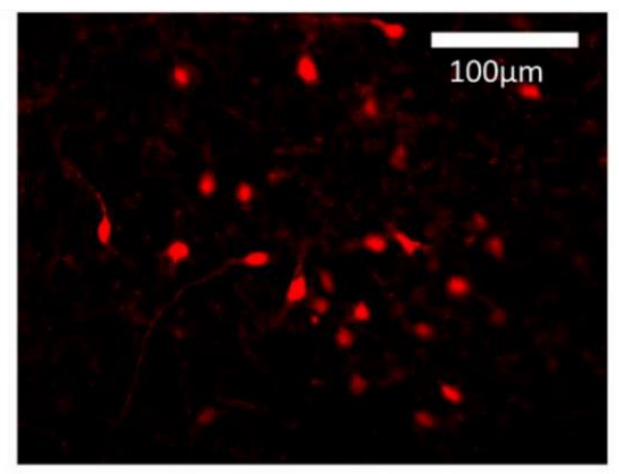

Figure 2. A, torpor TRAP protocol for TRAP2:Ai14 mice. Calorie restricted TRAP:Ai14 mice receive 4-hydroxytamoxifen (4-OHT) at ZT7, those that enter torpor following the injection are compared with those that do not. B, Counts of tdTomato-positive cells by region of interest in calorie-restricted mice that entered torpor following 4-OHT (red) or did not enter torpor following 4-OHT (blue). Torpor was associated with higher numbers of 'TRAPed' cells in the DMH and POA compared to animals that did not enter torpor. RM ANOVA comparing TRAPed cell count in mice that entered torpor with those that did not for each of the four regions of interest. Main effect for torpor vs no torpor, $p<0.05$, main effect for region of interest, $p<0.0001$, significant torpor vs no torpor by region of interest interaction, $p<0.05$. Holm-Sidak's multiple comparisons test, ${ }^{* *}$ indicates $p<0.01,{ }^{*}$ indicates $p<0.05$. Data shown are mean and standard deviation. C, coronal section showing POA neurons; $\mathrm{D}, \mathrm{DMH}$ and Arc neurons; $\mathrm{E}, \mathrm{PH}$ neurons. $\mathrm{F}$, sagittal schematic showing corresponding anterior-posterior location of coronal sections $C, D$, and $E$; G, high magnification image showing DMH torpor-active neurons expressing td-Tomato. Abbreviations: aca, anterior commissure (anterior part); A14, A14 dopamine cells; Arc, arcuate nucleus; DHA, dorsal hypothalamic area; DMH, dorsomedial hypothalamus; MPO, medial preoptic nucleus; MPA, medial preoptic area; PH, posterior hypothalamic nucleus; POA, preoptic area; VLPO, ventrolateral preoptic nucleus; $3 \mathrm{~V}$, third ventricle. $\mathrm{n}=5$ female mice per group. 
that did not enter torpor (DMH mean difference 185.6 TRAPed neurons, confidence interval (CI) 76 - 295, POA mean difference 135 TRAPed neurons, Cl 26 - 245). In contrast, the arcuate and posterior hypothalamus did not show significant differences between Torp+ and Torp- mice (see figure $2 \mathrm{~B}$ ). These differences in activity in the POA and DMH were not due to greater weight loss in the mice that entered torpor, indicating that the increased activity related to the occurrence of torpor per se rather than a response to the degree of calorie deficit (mean weight loss Torp $+2.6 \pm$ $0.7 \mathrm{~g}$, versus $2.4 \pm 0.3 \mathrm{~g}$ Torp-, $t(8)=0.66, p=0.46$; percentage weight loss Torp $+11.6 \pm 2.3 \%$ versus $11.0 \pm 1.2 \%$ Torp- $t(8)=0.57, p=0.58)$.

\section{Chemoactivation of torpor-TRAPed DMH neurons}

To test whether neurons within the DMH play a causal role in torpor, we targeted expression of the excitatory DREADD hM3Dq to neurons in the DMH that were active during torpor. Nine TRAP2 mice had injection of the Cre-dependent excitatory DREADD AAV (AAV2-hSyn-DIO-hM3DqmCherry) into the dorsomedial hypothalamus, followed by five days of calorie restriction with 4$\mathrm{OHT}$ injection on day five (see figure $3 \mathrm{~A}$ ). Of these mice, seven entered torpor following 4-OHT administration and were included in subsequent experiments (Torp+hM3Dq).

Chemoactivation of these neurons by injection of CNO to fed mice did not trigger torpor, nor did it alter surface temperature (see figure $3 \mathrm{~B}$ ). However, CNO delivered each day at ZT7, to calorie restricted mice, increased the probability of the mice entering torpor over five days (see figure 3CG). In a cross-over study design with CNO or saline, torpor emerged after fewer days of calorie restriction when mice received CNO compared to saline $(2.4 \pm 1.0$ versus $4.6 \pm 1.1, p<0.05)$. There were more torpor bouts per mouse on the $\mathrm{CNO}$ arms compared to the saline arms $(3.6 \pm 1.0$ versus 1.4 \pm 1.1 bouts, $p<0.05)$. All Torp $+\mathrm{hM} 3 \mathrm{Dq}$ mice entered torpor at least once when receiving CNO during five days of calorie restriction. Two mice did not enter torpor at all after completing five days calorie restriction when receiving saline.

When mice received $\mathrm{CNO}$ during the five days of calorie restriction, torpor bouts were longer than in the arms when they received saline $(F(1,6)=8.14, p<0.05)$. Likewise, during calorie restriction trials in which mice were given $\mathrm{CNO}$, the nadir temperature reached was lower than when they were given saline $(F(1,6)=12.2, p<0.05)$.

The weights of mice on entry into the CNO vs saline arm of the trial were not different $(24.7 \pm 2.7 \mathrm{~g}$ versus $24.7 \pm 2.7 \mathrm{~g}$ respectively, mean difference $-0.014 \mathrm{~g}$, paired $t(6)=0.049, p=0.96)$. Hence, the increased propensity to enter torpor observed with chemoactivation of hM3Dq-DMH-torpor-TRAP 
A

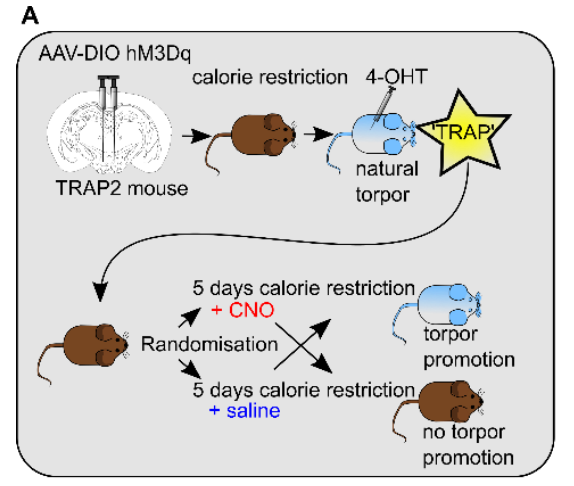

B

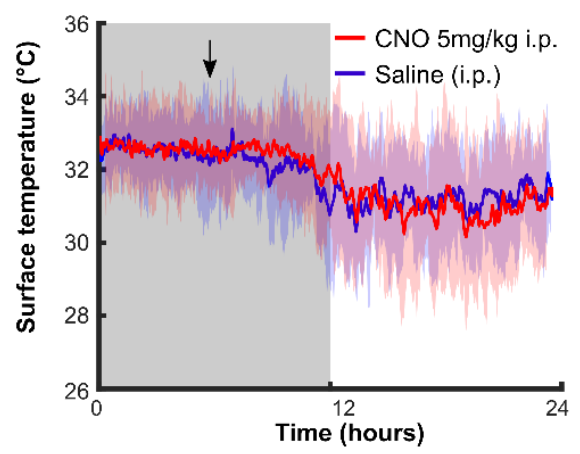

C
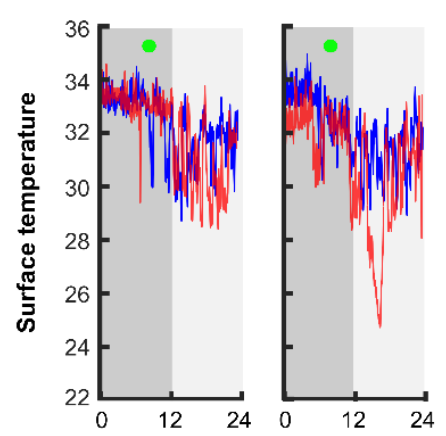

2

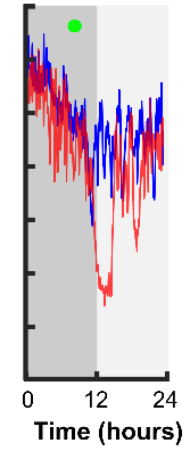

3

Consecutive days
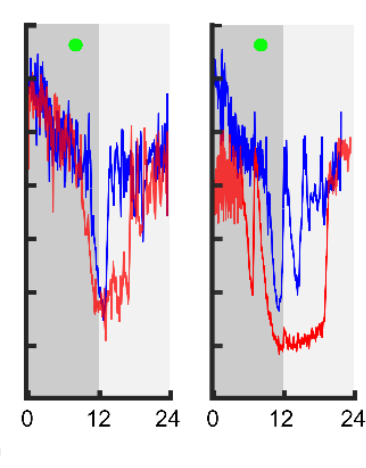

5
D
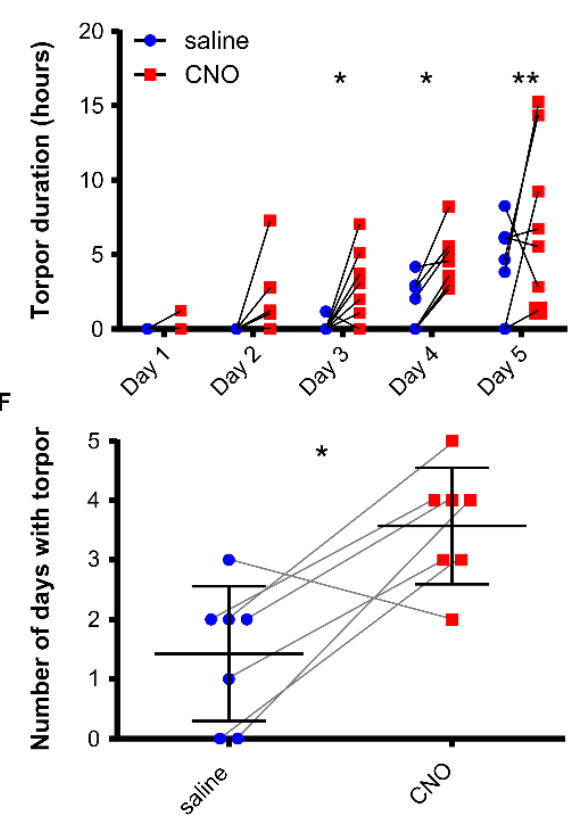

E
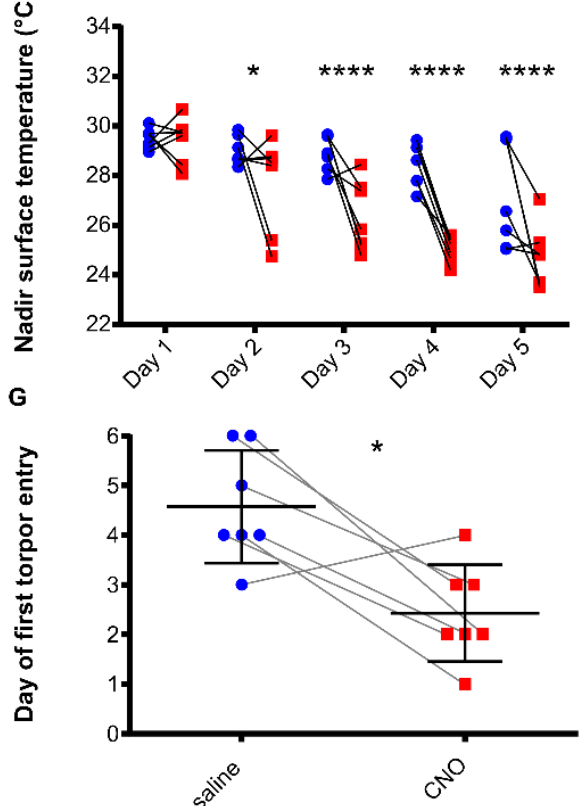

Figure 3. Chemoactivating DMH torpor-TRAPed neurons promotes, prolongs, and deepens torpor bouts in calorie restricted mice. A, $\mathrm{DMH}$ injection and torporTRAP protocol. B, CNO does not trigger torpor in $\mathrm{DMH}$ torpor-TRAPed mice that are not calorie restricted $(n=7$ DMH torpor-TRAPed female mice, shaded area represents 95\% Cl). C, example plot showing surface temperature in calorie restricted $\mathrm{DMH}$ torpor-TRAPed mice receiving CNO (red) or saline (blue) at 7 hours after lights off (green marker). D CNO given to calorie restricted DMH torporTRAPed mice increases the total time spent in torpor and decreases the nadir surface temperature reached $(D \& E$, respectively. 2-way repeated measures ANOVA, significant main effect for CNO vs saline trials, $p<0.05$ for both torpor duration and nadir temperature. Holm-Sidak's multiple comparisons test $*, * *, * *, * * *, \quad$ indicate significant difference between CNO and saline on individual day at $p<0.05,0.01,0.001$, 0.0001 , respectively). CNO increased the total number of days in which torpor occurred, and resulted in torpor occurring after fewer days of calorie restriction $(F \& G$, respectively. $*$ Indicates Wilcoxon matched-pairs signed rank test or paired $t$ test, $p<0.05$ ). $\mathrm{n}=7$ female mice. 
bioRxiv preprint doi: https://doi.org/10.1101/2021.09.05.458994; this version posted September 6, 2021. The copyright holder for this preprint (which was not certified by peer review) is the author/funder, who has granted bioRxiv a license to display the preprint in perpetuity. It is made available under aCC-BY-ND 4.0 International license.

A

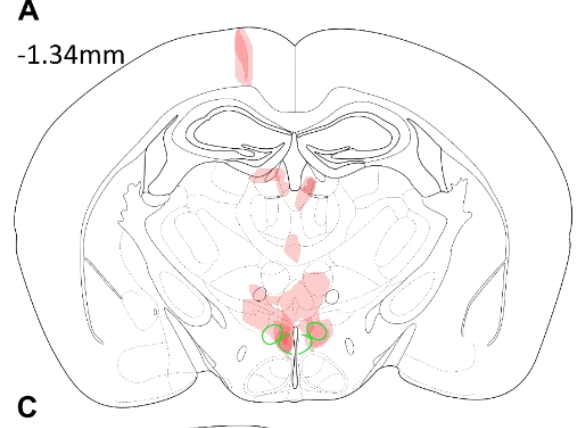

$-1.82 \mathrm{~mm}$

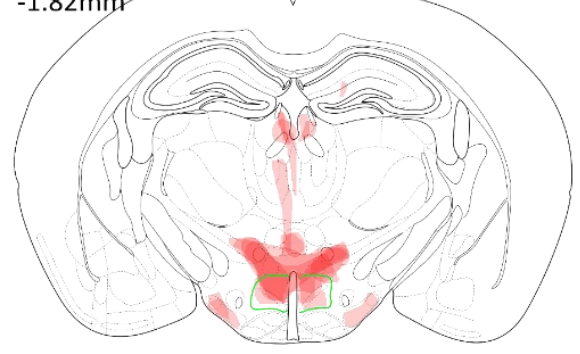

B

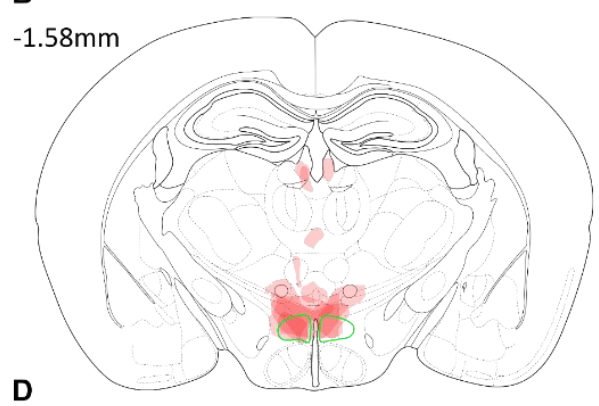

$-2.06 \mathrm{~mm}$

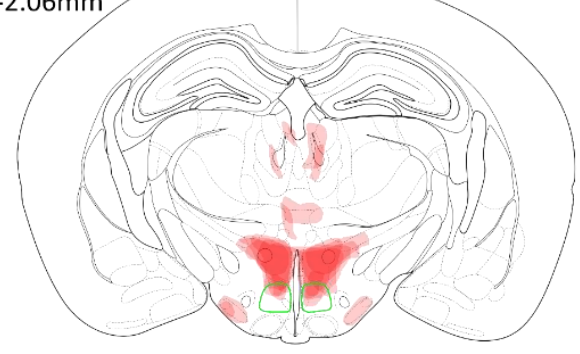

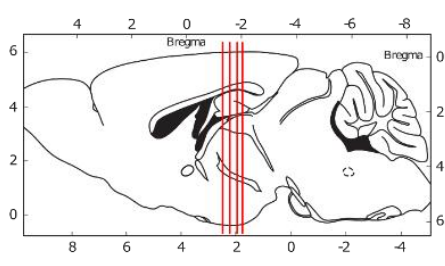

Key

$1 / 7$ animals

$2 / 7$ animals

$3 / 7$ animals

- $4 / 7$ animals

$5 / 7$ animals

$6 / 7$ animals

7/7 animals

$\checkmark$ Dorsomedial hypothalamus
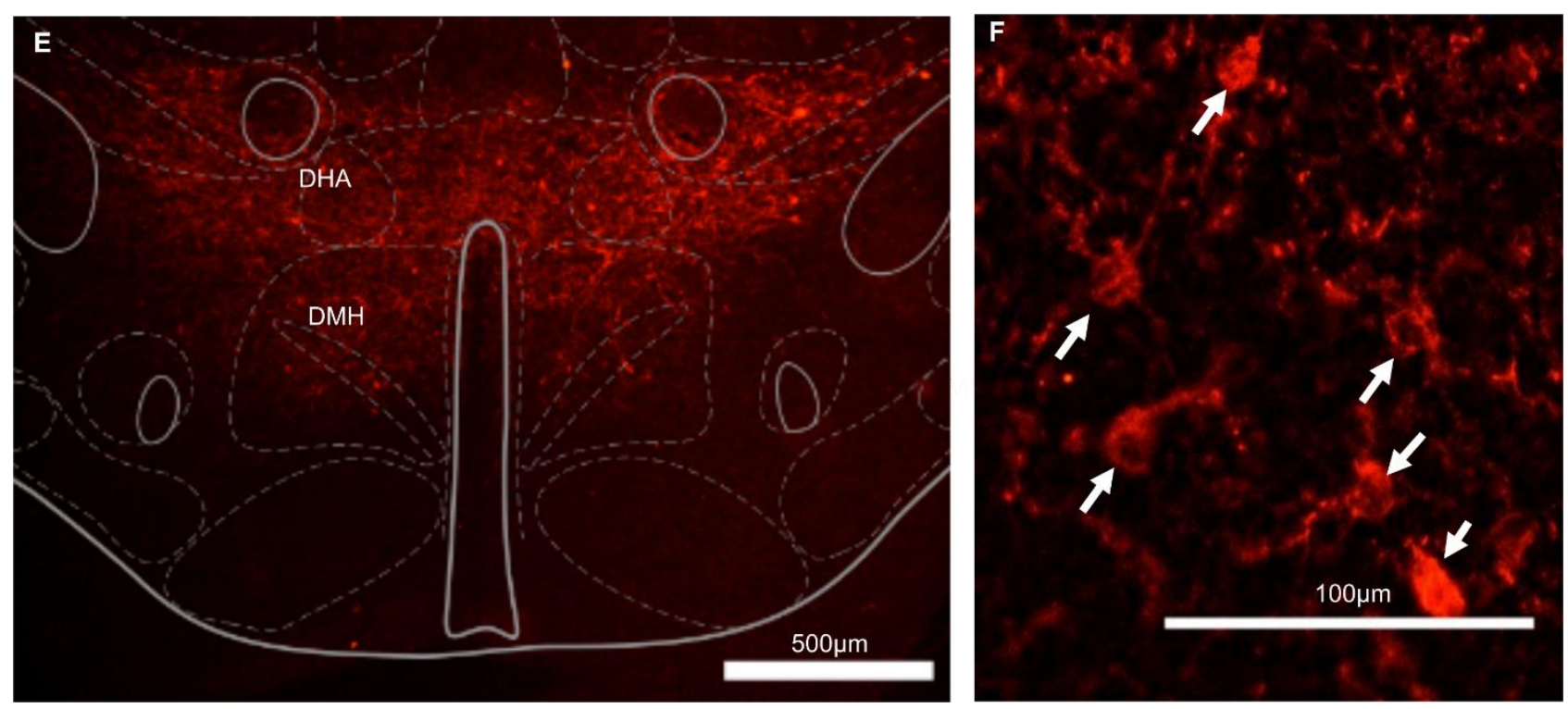

Figure 4. A-D, Mapped extent of mCherry-labelled cell bodies, indicating TRAPed cells expressing hM3DGq. TRAPed cells were observed in the dorsomedial hypothalamus ( $\mathrm{DMH}$, marked in green) and dorsal hypothalamic area (DHA) of all mice ( $(n=7$ female DMH torpor-TRAP mice). Injection tracts visible in A at bregma $-1.34 m m$, resulting in TRAPed cells in the cortex. Two mice also showed mCherry labelled cell bodies in the medial tuberal nucleus (visible in C \& D). $\mathrm{E} \& \mathrm{~F}$, mCherry labelling (red) indicating DREADD expression in TRAPed cells in an hM3Dq-DMH-TRAP mouse. Expression in the dorsomedial hypothalamus (DMH) and dorsal hypothalamic area (DHA). Immunohistochemistry performed labelling the mCherry component of the hM3Dq-mCherry fusion protein. F, Arrows indicate mCherry labelled neuron cell bodies. $15 \mu \mathrm{m}$ coronal sections. 
mice was not due to systematic differences in their weights. Torpor bouts tended to emerge at higher body weights on trials in which mice received CNO compared to saline, although this trend did not reach statistical significance (mean of differences CNO versus saline $1.01 \mathrm{~g}, p=0.098$ ).

\section{Chemoinhibition of torpor-TRAPed DMH neurons}

Twelve TRAP2 mice underwent injection of the Cre-dependent inhibitory DREADD (pAAV2-hSynDIO-hM4Di-mCherry) into the dorsomedial hypothalamus followed by five days of calorie restriction with 4-OHT injection on day five. Of these mice, six entered torpor following 4-OHT administration and were included (Torp+ hM4Di). These were evaluated for inhibition of torpor in response to CNO compared to saline.

Chemoinhibition of torpor-TRAPed DMH neurons in calorie restricted mice did not reduce the total number of days in which torpor occurred $(0.8 \pm 1.2$ (CNO) versus $1.7 \pm 1.2$ bouts (saline), paired $t(6)$ $=1.05, p=0.34)$. Nor did it delay the onset of torpor across the five days of calorie restriction (torpor appeared on day 5.2 \pm 1.2 (CNO) versus day $4.3 \pm 1.2$ days (saline), paired $t(5)=1.05, p$ $=0.34$ ) (see figure 5 ).

There were no differences between calorie restriction trials in which mice received CNO compared to when they received saline, in terms of time spent in torpor or nadir surface temperature reached $(F(1,5)=1.37, p=0.29$ and $F(1,5)=1.06, p=0.35$, respectively). Mouse weight on entry into the CNO arms was not different to that in the saline arms of the trial $(25.5 \pm 1.9 \mathrm{~g}$ versus 25.2 $\pm 2.1 \mathrm{~g}$ respectively, paired $t(5)=0.73, p=0.5$ ). Hence, activating inhibitory DREADDs in Torp+ hM4Di mice did not alter the expression of torpor during five days of calorie restriction. However, torpor bouts tended to emerge at lower body weights on trials in which mice received CNO compared to saline, consistent with a reduced propensity to torpor, although this trend did not reach statistical significance (mean of differences CNO versus saline $-0.85 g, p=0.20$ ).

\section{Controls}

Five mice underwent hM3Dq vector injection into the DMH followed by 4-OHT at ZT7 with free access to food in the homecage, 'Homecage $\mathrm{hM} 3 \mathrm{Dq}^{\prime}$. They were then entered into the randomised, crossover design, calorie restriction trial receiving CNO then saline or vice versa. Again, chemoactivation of DMH neurons TRAPed in the fed homecage condition had no effect on torpor, in terms of the day of first torpor bout appearance (4 [1.5-5.5] CNO, versus 3 [2-3], $p=0.5$, saline), total number of torpor bouts across five days calorie restriction ( $2.8 \pm 1.8$ versus $3.2 \pm 0.84$, 

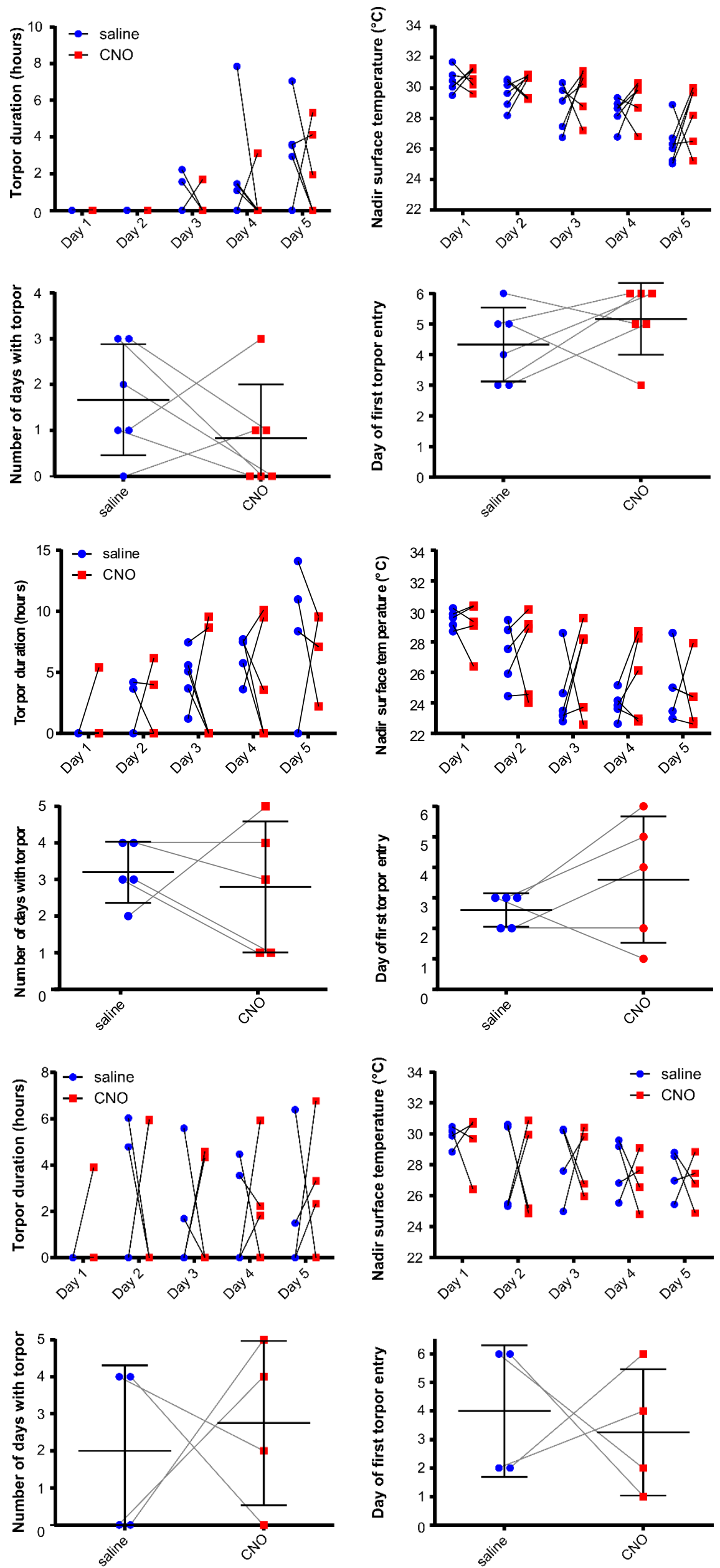

Figure 5. Chemoinhibition of $\mathrm{DMH}$ torpor-TRAPed DMH neurons does not affect torpor in calorie restricted mice. CNO did not affect the duration of torpor bouts, or nadir surface temperature reached (2-way repeated measures ANOVA, no main effect for CNO vs saline across all days, with Fisher's least significant difference test for CNO versus saline at each day of calorie restriction for each measure, $p>0.05$ throughout). CNO did not affect the total number of days in which torpor occurred, nor the first day on which torpor occurred during five days of calorie restriction (paired $t$-test, $p>0.05$ ). $\mathrm{n}$ $=6$ female mice.

Figure 6. CNO administration does not affect torpor in $\mathrm{DMH}$ homecageTRAPed mice. CNO did not affect the duration of torpor bouts, or the nadir surface temperature reached (2-way repeated measures ANOVA, no main effect for $\mathrm{CNO}$ vs saline across all days, with Fisher's least significant difference test for CNO versus saline at each day of calorie restriction for each measure, $p$ $>0.05$ throughout). CNO did not affect the total number of days in which torpor occurred, nor the first day on which torpor occurred during five days of calorie restriction (paired $t$-test, $p>0.05$ ). $\mathrm{n}=5$ female mice.

Figure 7. $\mathrm{CNO}$ administration does not affect torpor in wild type calorie-restricted mice. CNO did not affect the duration of torpor bouts, or the nadir surface temperature reached (2-way repeated measures ANOVA, no main effect for CNO vs saline across all days, with Fisher's least significant difference test for CNO versus saline at each day of calorie restriction for each measure, $p>0.05$ throughout). CNO did not affect the total number of days in which torpor occurred, nor the first day on which torpor occurred during five days of calorie restriction (paired $t$-test, $p>0.05$ ). $\mathrm{n}=4$ female mice. 
$p=0.69)$, daily nadir surface temperature $(p=0.48)$, or daily torpor bout duration $(p=0.83)$ (figure

6) (see figure 6).

CNO injection had no effect on torpor in wild type calorie restricted control mice $(n=4)$, in terms of the day of first torpor bout appearance (3 [0.5-4.75] versus $2[0-4], p=0.75)$, total number of torpor days in which torpor occurred across the five days calorie restriction (3 [1.25-5.5] versus 4 [2-6], $p=0.75)$, daily nadir surface temperature $(p=0.85)$, or daily torpor bout length $(p=0.78)$ (see figure 7).

\section{Discussion}

The data presented here indicate that both the $\mathrm{DMH}$ and the POA contain neurons that are active in the period leading up to a torpor bout and that chemoactivation of DMH torpor-TRAPed neurons increases the probability, as well as the depth and the duration of torpor, in calorie restricted mice.

The POA has been implicated in the induction of torpor, or torpor-like states in mice (Hrvatin et al., 2020; Takahashi et al., 2020; Zhang et al., 2020). Previous studies have also identified that the DMH contains neurons that express c-fos around the time of torpor (Hitrec et al., 2019; Hrvatin et al., 2020), and several studies have demonstrated that projections from the POA to the DMH can decrease body temperature (Song et al., 2016; Zhao et al., 2017; Takahashi et al., 2020). However, a precise role for the $\mathrm{DMH}$ in the control of torpor, rather than a homeostatic thermoregulatory, or counter-regulatory role has not been established. Here, by chemogenetically reactivating specifically those DMH neurons that previously expressed c-fos around the time of torpor, it has been possible to show that they do indeed contribute to the generation of torpor.

Chemoactivation of DMH torpor-TRAPed neurons did not suppress body temperature after single doses of CNO delivered to mice with free access to food. This suggests that the TRAPed neurons play a specific role in promoting torpor under calorie restricted conditions, but that they are not sufficient to trigger it in the fed state. They likely form part of a chorus of signals that indicate negative energy balance and the need to engage torpor. If on the other hand, the TRAPed neurons were simply part of a thermoregulatory circuit that inhibits thermogenesis under 'normal' homeostatic conditions, then one might expect chemoactivation to produce a physiological response that is independent of calorie restriction.

Whether there is a single group of neurons capable of inducing torpor, i.e. a torpor 'master switch' remains unknown, although emerging evidence places the POA as a potential candidate (Hrvatin 
et al., 2020; Takahashi et al., 2020; Zhang et al., 2020). The observation of torpor-promoting effects following chemoactivation of DMH-torpor-TRAP mice supports the hypothesis that a network of regions contributes to torpor induction. The DMH would then be considered one part of this network. If the POA is capable of triggering torpor, then the DMH might form part of the afferent signal to the POA, or it might modulate the descending efferent signals from the POA.

Another question is whether torpor is triggered, maintained, and terminated by the activity of a single population of neurons, or whether different populations are each responsible for timing the different phases. The observation that chemoactivating DMH torpor-TRAPed neurons increases bout duration as well as increasing the likelihood of torpor occurring hints that torpor may be induced and maintained by the same population of neurons. If, on the other hand, the effect of chemoactivation of DMH torpor-TRAPed neurons was to increase the probability but not the duration of torpor (or vice versa), then this would support the hypothesis that these phases of torpor are governed by distinct neuronal populations.

The finding that activation of neurons with in the $\mathrm{DMH}$ contributes to torpor induction - a physiological response characterised by suppression of thermogenesis - is particularly interesting. Within the framework of our current understanding of hypothalamic thermoregulatory circuits, the $\mathrm{DMH}$ generally emerges as a driver rather than inhibitor of thermogenesis, at least in rodents (Rezai-Zadeh et al., 2014; Zhao et al., 2017; Morrison and Nakamura, 2019). Under normal physiological circumstances in rodents housed in sub-thermoneutral conditions, the $\mathrm{DMH}$ is tonically active, driving the activity of sympathetic premotor neurons within the raphe pallidus, which in turn drive brown adipose tissue (BAT) thermogenesis. Body temperature is thus determined by the balance of activity of the projection from $\mathrm{DMH}$ to raphe, versus inhibitory inputs to the $\mathrm{DMH}$ from regions that respond to increases in skin, visceral, or hypothalamic temperature, such as the preoptic area (Cao, Fan and Morrison, 2004; DiMicco and Zaretsky, 2007). In contrast, the DMH neurons TRAPed during torpor in this study contribute to the suppression of thermogenesis, supporting a more complex and bi-directional role for the $\mathrm{DMH}$ in temperature regulation (Saper and Machado, 2020).

This raises the question of what is the phenotype of these $\mathrm{DMH}$ neurons? One might anticipate that they would be GABAergic, either acting as local inhibitory neurons or else projecting, for example to the raphe, to suppress BAT thermogenesis. Alternatively, a population of cholinergic neurons within the $\mathrm{DMH}$ respond to increases in ambient temperature, project to the raphe pallidus, and suppress BAT thermogenesis (Jeong et al., 2015b). Additional phenotypes within the 
DMH include NPY (Bi, Kim and Zheng, 2012), orexin (Nollet et al., 2011) and TRH (Chou et al., 2003), all of which contribute to energy balance and thermoregulation. Glia have been implicated in controlling food intake and energy balance (Argente-Arizón et al., 2017; MacDonald et al., 2019) and they express c-fos, hence they could also be TRAPed (Edling, Ingelman-Sundberg and Simi, 2007). However, the use of the synapsin promoter in the vectors described here means that they would be unlikely to express the viral transgene even if they were TRAPed.

Chemoinhibition of DMH torpor-TRAPed neurons did not prevent torpor induction in calorie restricted mice. It is worth noting that incomplete suppression of neuronal firing with the inhibitory DREADD, hM4Di, has been reported (Smith et al., 2016). Therefore, this result could be due to failure to inhibit these $\mathrm{DMH}$ neurons sufficiently to prevent their contribution to torpor. However, taken at face value, this finding suggests that while the torpor-TRAPed DMH neurons are capable of promoting torpor entry, their activity is not required for it. This conclusion is somewhat at odds with the model of torpor that emerges from previous studies, where optoactivation of a ' $Q$ neuron' projection from the POA to the $\mathrm{DMH}$ recapitulated the hypothermia of daily torpor in mice (Takahashi et al., 2020). This discrepancy could be explained by the fact that in the work presented here, we targeted specifically those $\mathrm{DMH}$ neurons that were active around the time of torpor. Crucially, this would not include neurons in the DMH whose activity was reduced in the period around torpor.

\section{Limitations}

If chemoactivation of DMH torpor-TRAPed neurons increased energy expenditure in already calorie restricted mice, then this might result in a greater energy deficit under CNO arms of the calorie restriction trials. It might then appear that chemoactivation was promoting torpor when in fact it was simply increasing energy deficit. However, the TRAP approach targeted neurons that were active just prior to natural torpor, when it is unlikely that neurons whose role is to increase energy expenditure would be active. Furthermore, the tendency for chemoactivation of DMH torpor-TRAPed neurons to result in torpor occurring at higher body weights again makes the interpretation that energy expenditure is increased less likely. Instead, it supports the hypothesis that the threshold for torpor has been reduced. The timing of 4-OHT delivery is critical. In our experiments, we aimed to deliver 4-OHT within three hours of torpor entry. This time period was chosen pragmatically in order to avoid disturbing the occurrence of torpor, while allowing TRAPing of the target populations. 
The timing of delivery of $4-\mathrm{OHT}$ is critical in determining the populations of neurons that are TRAPed. It is thought that neurons that are active within a period of approximately 3 hours either side of the injection will be TRAPed. We opted to deliver 4-OHT in anticipation of torpor in order to reduce the likelihood of arousing the mice and TRAPing arousal circuits. It is possible that this approach resulted in TRAPing neurons within the DMH that are not involved in torpor induction, and/or missed populations whose activity increased nearer to the point of torpor induction. Hence, it remains possible that delivery of $4-\mathrm{OHT}$ immediately prior to or even during torpor might TRAP a population of DMH neurons capable of inducing torpor in the absence of calorie restriction.

\section{Conclusions}

Our data indicate that neurons within the $\mathrm{DMH}$ promote torpor entry, and increase the duration and depth of torpor bouts in calorie restricted mice. Chemoactivating these neurons in fed mice neither triggered torpor nor altered thermoregulation, while chemoinhibiting them did not reduce torpor bout likelihood, duration, or depth. Hence, we hypothesise that the DMH is neither necessary nor sufficient for torpor entry, rather playing a modulatory promoting role. Important future work will phenotype these $\mathrm{DMH}$ neurons, and investigate the interaction between the POA and the $\mathrm{DMH}$ during torpor.

1. B. M. Barnes, Freeze avoidance in a mammal: Body temperatures below $0^{\circ} \mathrm{C}$ in an arctic hibernator. Science (80-. ). 244, 1593-1595 (1989).

2. G. Heldmaier, S. Ortmann, R. Elvert, Natural hypometabolism during hibernation and daily torpor in mammals. Respir. Physiol. Neurobiol. 141, 317-329 (2004).

3. S. Hrvatin, S. Sun, O. F. Wilcox, H. Yao, A. J. Lavin-Peter, M. Cicconet, E. G. Assad, M. E. Palmer, S. Aronson, A. S. Banks, E. C. Griffith, M. E. Greenberg, Neurons that regulate mouse torpor. Nature. 583, 115-121 (2020).

4. T. M. Takahashi, G. A. Sunagawa, S. Soya, M. Abe, K. Sakurai, K. Ishikawa, M. Yanagisawa, H. Hama, E. Hasegawa, A. Miyawaki, K. Sakimura, M. Takahashi, T. Sakurai, A discrete neuronal circuit induces a hibernation-like state in rodents. Nature. 583 (2020), doi:10.1038/s41586020-2163-6.

5. Z. Zhang, F. M. C. V. Reis, Y. He, J. W. Park, J. R. DiVittorio, N. Sivakumar, J. E. van Veen, S. 
Maesta-Pereira, M. Shum, I. Nichols, M. G. Massa, S. Anderson, K. Paul, M. Liesa, O. A. Ajijola, Y. Xu, A. Adhikari, S. M. Correa, Estrogen-sensitive medial preoptic area neurons coordinate torpor in mice. Nat. Commun. 11, 1-14 (2020).

6. Z. Zhao, W. Z. Yang, C. Gao, X. Fu, W. Zhang, Q. Zhou, W. Chen, X. Ni, J. K. Lin, J. Yang, X. H. Xu, W. L. Shen, A hypothalamic circuit that controls body temperature. Proc. Natl. Acad. Sci. U. S. A. 114, 2042-2047 (2017).

7. C. L. Tan, E. K. Cooke, D. E. Leib, Y. C. Lin, G. E. Daly, C. A. Zimmerman, Z. A. Knight, WarmSensitive Neurons that Control Body Temperature. Cell. 167, 47-59.e15 (2016).

8. S. Bi, Y. J. Kim, F. Zheng, Dorsomedial hypothalamic NPY and energy balance control. Neuropeptides. 46, 309-314 (2012).

9. L. Yang, K. A. Scott, J. Hyun, K. L. Tamashiro, N. Tray, T. H. Moran, S. Bi, Role of dorsomedial hypothalamic neuropeptide $Y$ in modulating food intake and energy balance. J. Neurosci. 29, 179-190 (2009).

10. J. H. Jeong, D. K. Lee, C. Blouet, H. H. Ruiz, C. Buettner, S. Chua, G. J. Schwartz, Y. H. Jo, Cholinergic neurons in the dorsomedial hypothalamus regulate mouse brown adipose tissue metabolism. Mol. Metab. 4, 483-492 (2015).

11. R. A. Piñol, S. H. Zahler, C. Li, A. Saha, B. K. Tan, V. Škop, O. Gavrilova, C. Xiao, M. J. Krashes, M. L. Reitman, B rs3 neurons in the mouse dorsomedial hypothalamus regulate body temperature, energy expenditure, and heart rate, but not food intake. Nat. Neurosci. 21 (2018), pp. 1530-1540.

12. J. J. Gooley, A. Schomer, C. B. Saper, The dorsomedial hypothalamic nucleus is critical for the expression of food-entrainable circadian rhythms. Nat. Neurosci. 9, 398-407 (2006).

13. M. Mieda, S. C. Williams, J. A. Richardson, K. Tanaka, M. Yanagisawa, The dorsomedial hypothalamic nucleus as a putative food-entrainable circadian pacemaker. Proc. Natl. Acad. Sci. 103, 12150-12155 (2006).

14. T. C. Chou, T. E. Scammell, J. J. Gooley, S. E. Gaus, C. B. Saper, J. Lu, Critical Role of Dorsomedial Hypothalamic Nucleus in a Wide Range of Behavioral Circadian Rhythms. J. Neurosci. 23, 10691-10702 (2003).

15. T. Hitrec, M. Luppi, S. Bastianini, F. Squarcio, C. Berteotti, V. Lo Martire, D. Martelli, A. 
Occhinegro, D. Tupone, G. Zoccoli, R. Amici, M. Cerri, Neural control of fasting-induced torpor in mice. Sci. Rep. 9, 1-12 (2019).

16. L. A. DeNardo, C. D. Liu, W. E. Allen, E. L. Adams, D. Friedmann, L. Fu, C. J. Guenthner, M. Tessier-Lavigne, L. Luo, Temporal evolution of cortical ensembles promoting remote memory retrieval. Nat. Neurosci. 22, 460-469 (2019).

17. B. L. Roth, DREADDs for Neuroscientists. Neuron. 89, 683-694 (2016).

18. W. E. Allen, L. A. DeNardo, M. Z. Chen, C. D. Liu, K. M. Loh, L. E. Fenno, C. Ramakrishnan, K. Deisseroth, L. Luo, Thirst-associated preoptic neurons encode an aversive motivational drive. Science (80-. ). 357, 1149-1155 (2017).

19. L. Madisen, T. A. Zwingman, S. M. Sunkin, S. W. Oh, H. A. Zariwala, H. Gu, L. L. Ng, R. D. Palmiter, M. J. Hawrylycz, A. R. Jones, E. S. Lein, H. Zeng, A robust and high-throughput Cre reporting and characterization system for the whole mouse brain. Nat. Neurosci. 13, 133140 (2010).

20. S. J. Swoap, M. J. Gutilla, Cardiovascular changes during daily torpor in the laboratory mouse. Am. J. Physiol. - Regul. Integr. Comp. Physiol. 297, R769-74 (2009).

21. M. J. Krashes, S. Koda, C. Ye, S. C. Rogan, A. C. Adams, D. S. Cusher, E. Maratos-Flier, B. L. Roth, B. B. Lowell, Rapid, reversible activation of AgRP neurons drives feeding behavior in mice. J. Clin. Invest. 121, 1424-1428 (2011).

22. V. van der Vinne, M. J. Bingaman, D. R. Weaver, S. J. Swoap, Clocks and meals keep mice from being cool. J. Exp. Biol. 221, jeb179812 (2018).

23. C. on A. N. Subcommittee on Laboratory Animal Nutrition, N. R. C. Board on Agriculture, Nutrient Requirements of Laboratory Animals, (National Academies Press, Washington, D.C., 1995; http://www.nap.edu/catalog/4758).

24. K. B. J. Franklin, G. Paxinos, The Mouse Brain in stereotaxic coordinates (2007).

25. C. J. Guenthner, K. Miyamichi, H. H. Yang, H. C. Heller, L. Luo, Permanent genetic access to transiently active neurons via TRAP: Targeted recombination in active populations. Neuron. 78, 773-784 (2013).

26. K. Song, H. Wang, G. B. Kamm, J. Pohle, F. de C. Reis, P. Heppenstall, H. Wende, J. Siemens, The TRPM2 channel is a hypothalamic heat sensor that limits fever and can drive 
hypothermia. Science (80-. ). 353, 1393-1398 (2016).

27. K. Rezai-Zadeh, S. Yu, Y. Jiang, A. Laque, C. Schwartzenburg, C. D. Morrison, A. V Derbenev, A. Zsombok, H. Münzberg, Leptin receptor neurons in the dorsomedial hypothalamus are key regulators of energy expenditure and body weight, but not food intake. Mol. Metab. 3, 681-93 (2014).

28. W. H. Cao, W. Fan, S. F. Morrison, Medullary pathways mediating specific sympathetic responses to activation of dorsomedial hypothalamus. Neuroscience. 126, 229-240 (2004).

29. J. A. DiMicco, D. V Zaretsky, The dorsomedial hypothalamus: a new player in thermoregulation. Am. J. Physiol. Integr. Comp. Physiol. 292, R47-R63 (2007).

30. C. B. Saper, N. L. S. Machado, Flipping the switch on the thermoregulatory system Atomic forces mapped out by lasers. Nature (2020).

31. J. H. Jeong, D. K. Lee, C. Blouet, H. H. Ruiz, C. Buettner, S. Chua, G. J. Schwartz, Y. H. Jo, Cholinergic neurons in the dorsomedial hypothalamus regulate mouse brown adipose tissue metabolism. Mol. Metab. 4, 483-492 (2015).

32. M. Nollet, P. Gaillard, F. Minier, A. Tanti, C. Belzung, S. Leman, Activation of orexin neurons in dorsomedial/perifornical hypothalamus and antidepressant reversal in a rodent model of depression. Neuropharmacology. 61, 336-346 (2011).

33. A. J. MacDonald, F. E. Holmes, C. Beall, A. E. Pickering, K. L. J. Ellacott, Regulation of food intake by astrocytes in the brainstem dorsal vagal complex. Glia, 1-14 (2019).

34. P. Argente-Arizón, S. Guerra-Cantera, L. M. Garcia-Segura, J. Argente, J. A. Chowen, Glial cells and energy balance. J. Mol. Endocrinol. 58, R59-R71 (2017).

35. Y. Edling, M. Ingelman-Sundberg, A. Simi, Glutamate activatesc-fos in glial cells via a novel mechanism involving the glutamate receptor subtype mGlu5 and the transcriptional repressor DREAM. Glia. 55, 328-340 (2007).

36. K. S. Smith, D. J. Bucci, B. W. Luikart, S. V. Mahler, DREADDs: Use and application in behavioral neuroscience. Behav. Neurosci. 130, 137-155 (2016). 
bioRxiv preprint doi: https://doi.org/10.1101/2021.09.05.458994; this version posted September 6, 2021. The copyright holder for this preprint (which was not certified by peer review) is the author/funder, who has granted bioRxiv a license to display the preprint in perpetuity. It is made available under aCC-BY-ND 4.0 International license. 